\title{
Mixed acinar-endocrine carcinoma of pancreas: a case report and brief review of the literature
}

This article was published in the following Dove Press journal:

OncoTargets and Therapy

3 July 2015

Number of times this article has been viewed

\author{
Zhenzhen Liu',2 \\ Chengyong Dong', \\ Chengye Wang ${ }^{1,2}$ \\ Qinlong Liu' \\ Deguang Sun' \\ Liming Wang'
}

'Department of General Surgery, The Second Affiliated Hospital of Dalian Medical University, ${ }^{2}$ Dalian Medical University, Dalian, Liaoning Province, People's Republic of China
Correspondence: Liming Wang Department of General Surgery, The Second Affiliated Hospital of Dalian Medical University, No. 467 Zhongshan Road, Shahekou District, Dalian, Liaoning Province, II6027, People's Republic of China

Email wangbcc259@163.com
Abstract: Mixed acinar-endocrine carcinoma (MAEC) of the pancreas is a rare entity. We present a 65-year-old Chinese female who was admitted with jaundice and nagging epigastric pain with intermittent diarrhea for 1 month. She eventually underwent abdominal magnetic resonance imaging, which showed an $8 \times 6 \mathrm{~cm}$ mass in the head of the pancreas and showed two abnormal lesions in the liver simultaneously. MAEC of the pancreas with synchronous hepatic metastasis was confirmed with immunohistochemistry after Whipple operation and hepatic partial resection of the lesions. Postoperative recovery of this patient was uneventful, and no evidence of recurrence or metastasis was observed after 12 months of follow-up. MAEC of pancreas is thought to be extremely rare and lack of typical clinical symptoms. The prognosis is poor overall, but early detection with complete resection may be beneficial to patients.

Keywords: acinar cell carcinoma, neuroendocrine carcinoma of pancreas, neuroendocrine carcinoma, pancreatic neoplasms

\section{Introduction}

Mixed acinar-endocrine carcinoma (MAEC) of the pancreas is a rare entity, ${ }^{1}$ with 30 or so cases of pancreas MAEC reported in the English literature (Table S1). To date, its biological behaviors, the appropriate treatment modalities, and the overall prognosis remain unclear. Herein, we report a rare case of pancreas MAEC and present a brief literature review to contribute to the increased understanding of the clinical features of this disease.

\section{Case report}

The patient was a 65-year-old woman who presented with jaundice and nagging epigastric pain with intermittent diarrhea for 1 month. She denied fevers or chills, malaise, fatigue, or weight loss. She denied family history of pancreatic cancer and other cancers. She denied hepatitis B and other infectious diseases. Physical examination revealed that the sclera was mildly icteric and light tenderness could be felt under the xiphoid process, without rebound tenderness and muscle tension. There was a mass about $10 \mathrm{~cm} \times 7 \mathrm{~cm}$ around the navel, without a clear boundary between surrounding tissues, cystosolid, and no obvious abnormality was seen in the rest parts of the whole body. Laboratory tests revealed the following: aspartate aminotransferase (AST) 68.00 U/L (normal range 13-35 U/L); alanine transaminase (ALT) $138.00 \mathrm{U} / \mathrm{L}$ (normal range 7-40 U/L); total bilirubin $89.20 \mu \mathrm{mol} / \mathrm{L}$ (normal range $2-20 \mu \mathrm{mol} / \mathrm{L}$ ); direct bilirubin $72.21 \mu \mathrm{mol} / \mathrm{L}$ (normal range $0-6 \mu \mathrm{mol} / \mathrm{L}$ ); $\gamma$-glutamyltransferase ( $\gamma$-GGT) $948.00 \mathrm{U} / \mathrm{L}$ (normal range 7-45 U/L); alkaline phosphatase (ALP) 494.00 $\mathrm{U} / \mathrm{L}$ (normal range 50-135 U/L); and normal albumin, amylase, and glucose. Cancer antigen 19-9 (CA19-9), cancer antigen 125 (CA125), cancer antigen 153 (CA153), 

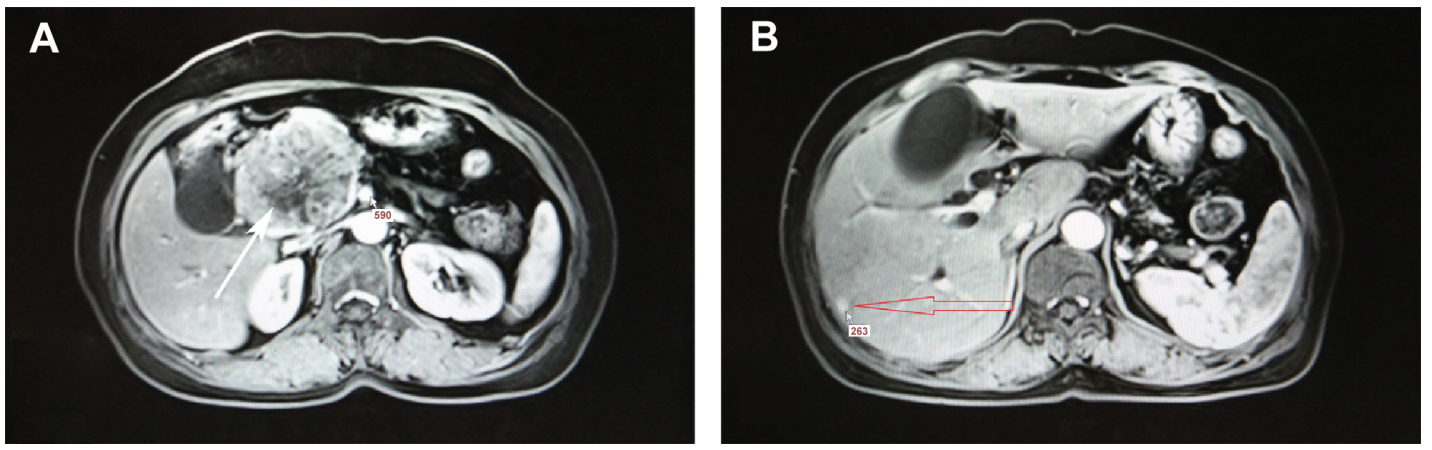

Figure I Abdominal enhanced magnetic resonance imaging with TI findings.

Notes: (A) An $8 \times 6 \mathrm{~cm}$ mass in the head of the pancreas (white arrow). (B) Liver metastasis from mixed acinar-endocrine carcinoma of the pancreas (red arrow).

and carcinoembryonic antigen (CEA) were normal. Alphafetoprotein (AFP) was $156.80 \mathrm{IU} / \mathrm{mL}$ (normal range $0.00-6.70 \mathrm{IU} / \mathrm{mL}$ ). Chromogranin A level was $136 \mathrm{U} / \mathrm{L}$ (normal range $0-95 \mathrm{U} / \mathrm{L}$ in our hospital) and pancreatic polypeptide level was $307 \mathrm{ng} / \mathrm{L}$ (normal range $105-175 \mathrm{ng} / \mathrm{L}$ ). The patient eventually underwent abdominal magnetic resonance imaging, which showed an $8 \times 6 \mathrm{~cm}$ mass in the head of the pancreas and showed two abnormal lesions about $2 \times 1 \mathrm{~cm}$ in the liver (Figure 1). Endoscopic ultrasound-guided fineneedle aspiration (FNA) of the mass was performed and its histopathology indicated pancreatic carcinoma. The patient then underwent pancreaticoduodenectomy (Whipple procedure) with tumor-free margins and negative lymph nodes. She underwent hepatic partial resection of the two lesions simultaneously. Pathological examination of the surgical specimen of pancreatic mass revealed a poorly circumscribed, elastic, soft, grayish-yellow section. Microscopically, the tumor cells had predominately solid and partly acinar morphology. The tumor measured $8 \mathrm{~cm}$ in the greatest dimension, extending into peripancreatic adipose tissue. It extended beyond the head of the pancreas but without the involvement of the celiac axis or the superior mesenteric vein or artery. Pathological examination of the surgical specimen of two liver abnormal lesions were confirmed as metastatic lesions of pancreatic mass. However, no regional lymph node metastasis was identified. Immunohistochemical staining of pancreatic mass was positive for trypsin and chymotrypsin, which indicated the tissue had an acinar component. Synaptophysin, chromogranin A, CD56, and neuron-specific enolase (NSE) were also positive for immunohistochemistry, suggesting that it had an endocrine component. Ki-67 had 10\% positivity. Each component exceeded $25 \%$ of the tumor, and pathological diagnosis of MAEC was established (Figure 2). Microscopically, the tumor cells of liver metastasis had partly solid and partly acinar morphology. Immunohistochemical staining of liver metastasis was positive for trypsin and weakly positive for synaptophysin, chromogranin A, and NSE (Figure 3). According to pathological analysis of primary MAEC of pancreas, the liver lesions showed a similar histological character to the primary pancreatic lesion, which suggested that the liver lesions derived from pancreatic metastatic lesions. After surgery, gastrin, glucagon, glucose, somatostatin, pancreatic polypeptide, vasoactive intestinal peptide, and 5-hydroxytryptamine (serotonin) were detected, and all of these indicators were within normal limits. This might explain why the patient did not have clinical symptoms of abnormal hormone secretion, which is common in pancreatic neuroendocrine tumors. Moreover, she also underwent an octreotide scan after surgery which showed no evidence of uptake. However, the patient refused chemotherapy but wanted to accept regular checks. During the 12 months of follow-up, no evidence of recurrence or metastasis was observed.

\section{Discussion}

The pancreas is composed of exocrine and endocrine gland components, with the exocrine part comprising ductal and acinar cells, whereas the endocrine part is made up of endocrine cells. Pancreatic acinar cell carcinomas (ACCs) are uncommon, accounting for less than $2 \%$ of all pancreatic neoplasms, ${ }^{1,2}$ while pancreatic neuroendocrine tumors are less than $3 \%$ of all pancreatic neoplasms, without a significant sex predilection. ${ }^{3}$ However, ACCs have been found to show both ductal and endocrine differentiation. ${ }^{1}$ Many researchers believe that MAEC of the pancreas is generally considered a variant of ACC. ${ }^{4}$ Pathological diagnosis of MAEC of the pancreas is based on these criteria: morphology of acinar and endocrine cells, and immunohistochemistry demonstrating both acinar markers (trypsin, chymotrypsin, lipase, and periodic acid-Schiff) and endocrine markers (chromogranin A, synaptophysin, CD56, NSE), at least $25 \%-30 \%$ for acinar markers and $25 \%-30 \%$ for endocrine 

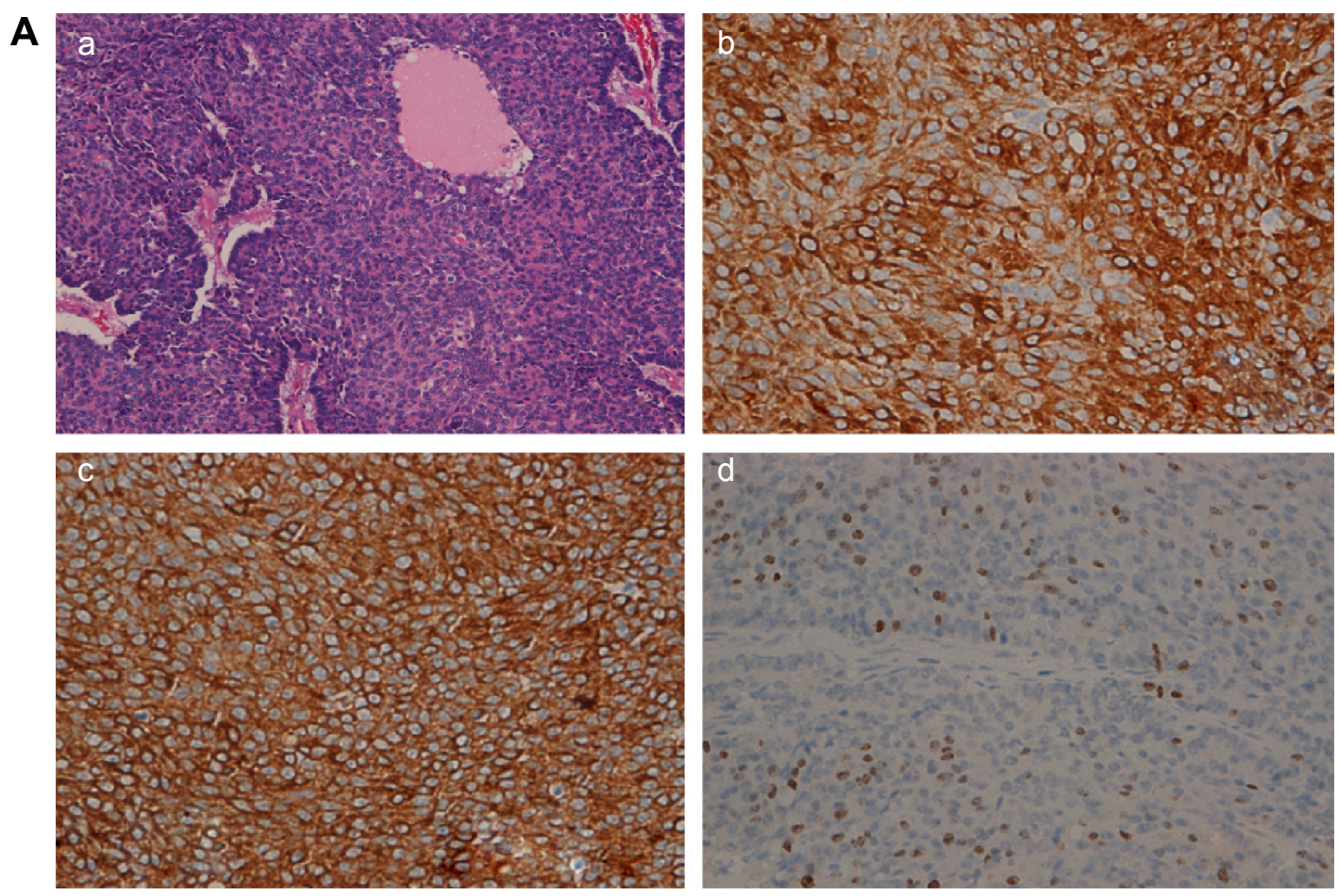

B
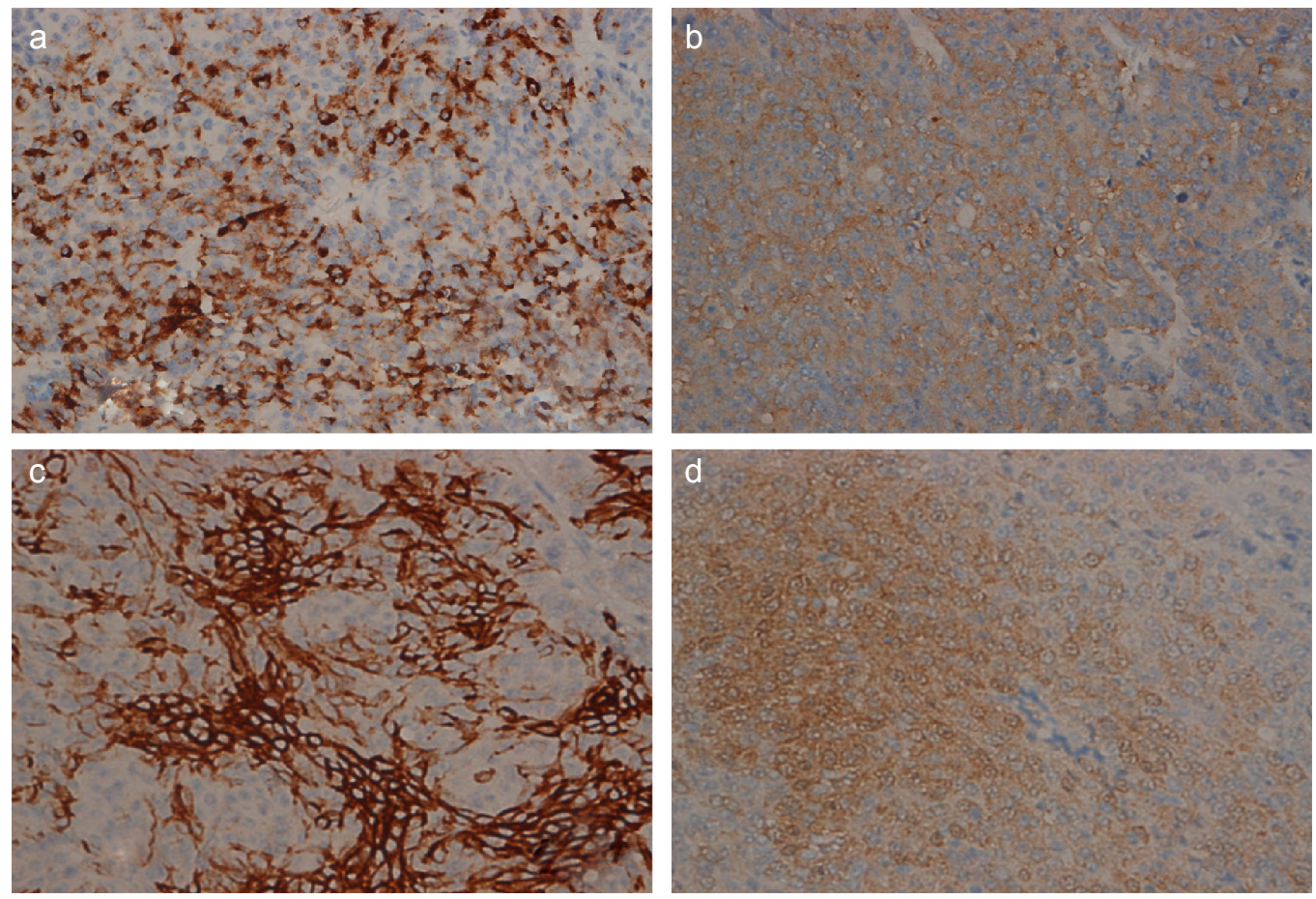

Figure 2 Histopathology of the pancreas tumor.

Notes: (A) (a) Hematoxylin-eosin image of the pancreas tumor (HEX200). (b) Immunohistochemistry of trypsin ( $\times 400$, Abcam). (c) Immunohistochemistry of chymotrypsin ( $\times 400$, Abcam). (d) Immunohistochemistry of Ki-67 ( $\times 400$, Abcam [Cambridge, MA, USA]). (B) (a) Immunohistochemistry of chromogranin A ( $\times 400$, Abcam) (b) Immunohistochemistry of synaptophysin ( $\times 400$, Abcam). (c) Immunohistochemistry of CD56 ( $\times 400$, Abcam). (d) Immunohistochemistry of neuron-specific enolase (NSE) $(\times 400$, Abcam).

markers. ${ }^{5,6} \mathrm{ACCs}$ of the pancreas have been known to express endocrine markers as well in up to one-third of the cases, which are usually limited to a few scattered cells. ${ }^{2,7}$ Although pancreatic endocrine neoplasms and ACC are distinct entities, their pathological and morphological appearances are sometimes extremely similar, and their components can be combined. ${ }^{8}$ When each component exceeds $25 \%$ of the tumor, the condition is defined as MAEC. ${ }^{6}$ However, little is known about MAEC because of its rarity. ${ }^{9}$ In MAEC, two different patterns of histopathologic structure have 

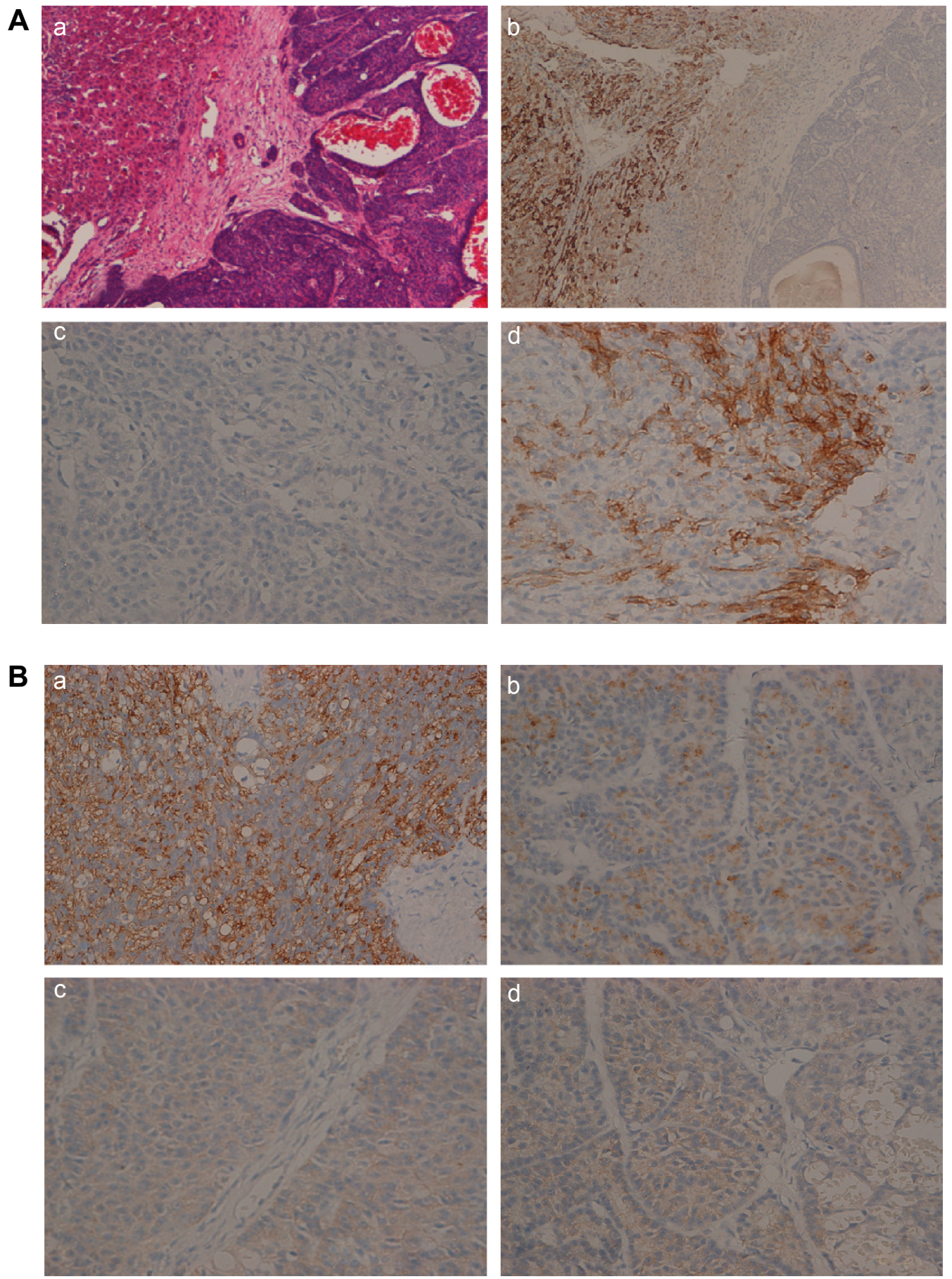

Figure 3 Histopathology of the liver metastasis.

Notes: (A) (a) Hematoxylin-eosin image of the liver metastasis (HEXI00). Left is adjacent cancerous tissue and right is the liver metastasis. (b) Immunohistochemistry of hepatocyte paraffin I (Hep Par I) ( $\times 100$, Dako). Adjacent cancerous tissue was positive for immunohistochemistry of Hep Par I and the liver metastasis was negative for the same. (c) The liver metastasis was negative for immunohistochemistry of Hep Par I ( $\times 400$, Dako Denmark A/S, Glostrup, Denmark). (d) Immunohistochemistry of CD56 $(\times 400, A b c a m)$. (B) (a) Immunohistochemistry of trypsin ( $\times 400, A b c a m)$, indicating that the tissue had an acinar component. (b) Immunohistochemistry of chromogranin A ( $\times 400$, Abcam). (c) Immunohistochemistry of synaptophysin $(\times 400$, Abcam). (d) Immunohistochemistry of neuron-specific enolase (NSE) ( $\times 400$, Abcam). Chromogranin A, synaptophysin, and NSE were weakly positive for immunohistochemistry, suggesting that the tissue of the liver metastasis had endocrine component.

been demonstrated. One is the coexistence of the acinar and endocrine components, and the other is their coexpression. ${ }^{10}$ The former consists of a histological segregation in the tumor differentiation. ${ }^{10}$ Each acinar and endocrine cellular component is entirely separate without intermingling.
The coexpression pattern consists of an admixture of both components, in which the neoplastic cells simultaneously show both acinar and endocrine differentiation..$^{10,11}$ In our case, histology of the tumor presented with both acinar and endocrine differentiation and the neoplastic cells diffusely 
expressed the acinar and endocrine markers (trypsin, chymotrypsin, chromogranin A, and synaptophysin), which indicated that tumor differentiation was towards both acinar and endocrine carcinomas.

MAEC of pancreas is an incredibly rare entity, ${ }^{1}$ with only about 30 or so cases in the published English literature (Table S1). Based on these reported cases, 21 of all 35 patients with confirmed MAEC of the pancreas were male and the male:female ratio was 1.5:1.0, suggesting that MAEC of the pancreas affects both sexes. However, male patients may be more commonly affected, which is similar to ACC. ${ }^{8}$ The notion that MAEC of the pancreas mostly affects female patients ${ }^{4,11}$ may not hold true, which agrees with Yu et al. ${ }^{5}$ However, this may be incidental due to the small number of MAEC cases that have been reported. MAEC of pancreas is most commonly located at the head (18/35) and is often in middle-aged individuals (mean age of 60.9 years, except the 89-year-old patient [maximum value] and 6 -year old patient [minimum value] were excluded when we calculated the mean age) (Table S1), which suggests MAECs of the pancreas should be considered in middleaged patients with pancreatic head solid mass. In our case, the patient was no exception. The tumor size varied and the mean size was $6.95 \mathrm{~cm}$ in the greatest dimension. Abdominal pain and mass are common clinical manifestations in MAEC, but they are not a specific sign. In fact, the variable clinical behaviors of MAEC of the pancreas lack a reliable indicator so that we can detect the tumor early. In addition, all patients but one who presented with Zollinger-Ellison syndrome ${ }^{12}$ did not exhibit hormone excess syndrome. Jaundice is relatively uncommon since MAEC is a well-circumscribed tumor and rarely invades surrounding tissue like the common bile duct, which could also be found in ACC. ${ }^{13}$ However, in our case, the patient was jaundiced because of tumor-mass effects.

The clinical diagnosis of MAEC of the pancreas remains a big challenge. The most common differential diagnoses of solid pancreatic mass are ductal carcinoma followed by neuroendocrine tumors. ${ }^{14,15}$ Other forms of solid pancreatic masses such as ACC, pancreatoblastomas, and solid pseudopapillary neoplasms are also easily confused. ${ }^{16,17}$ Therefore, it is not surprising that the preoperative clinical diagnosis does not correctly identify MAEC of the pancreas. ${ }^{15}$ In our case, we conducted the endoscopic ultrasound-guided FNA of the pancreatic mass and it suggested pancreatic carcinoma. This may suggest the FNA still has some limitations for making a definite diagnosis. ${ }^{18}$ Therefore, the diagnosis of pancreas MAEC is mainly made by pathological analysis of the tumor morphology and immunohistochemical staining.
To our knowledge, about 15 sporadic single-case reports published over the last decade have explicitly recorded the quantification of acinar/endocrine components so that MAEC of the pancreas diagnosis could be verified ${ }^{5}$ (Table S1). As is shown in Table S1, trypsin, chymotrypsin, lipase, and periodic acid-Schiff were used for immunohistochemistry of acinar markers, while chromogranin A and synaptophysin were for endocrine markers.

Due to the small number of cases of MAEC reported, no standardized management protocol has been established. However, based on cases of MAEC reported on the current literature (Table S1), it is generally agreed that surgery is the prime treatment for the cases with resectable tumor. ${ }^{2}$ Moreover, the large presenting tumor size and high $\mathrm{Ki}-67$ index suggest that MAEC of the pancreas could grow rapidly if untreated. There also have been reports of patients benefiting from tumor debulking surgery and local and systemic antiproliferative therapy. ${ }^{7}$ The survival time of MAEC patients after surgical resection of the primary tumor varies. It was reported that there were only 21 of 35 cases of pancreas MAEC recording the survival time after surgical resection. The median overall survival time is about 12 months when the 21 cases are analyzed by the life table method, and the 12-month cumulative survival rate is $85 \%$, as is shown in Table S2 and Figure S1. However, the overall survival of patients with ACC is reported to be from 18 to 19 months, ${ }^{19}$ which suggests that the prognosis of patients with MAEC shows lots of similarities to ACC. ${ }^{13}$ Moreover, the results of adjuvant chemotherapy and radiotherapy for MAEC have also been disappointing as well as ACC. Moreover, although many reports have shown that genetic alterations in the APC/ $\beta$-catenin pathway are common in ACC, we have little knowledge about the genetic alterations of MAEC since no researchers have reported it. Hence, we expect that the deep sequencing of MAEC can give us some hints about the genetic features of this rare tumor, which will definitely improve our understanding and treatment of this disease..$^{20}$

\section{Conclusion}

MAEC of the pancreas is an extremely rare tumor of the pancreas for which surgical resection remains a beneficial treatment. Patients need regular follow-up since it has a tendency of metastasis. There remains much uncertainty with MAEC because of the limited number of cases. Therefore, the accumulation of additional data from more cases is necessary to further elucidate this type of carcinoma and standardize optimal therapy.

\section{Acknowledgment}

Patient consent was obtained for this study. 


\section{Disclosure}

The authors report no conflicts of interest in this work.

\section{References}

1. Stelow EB, Shaco-Levy R, Bao F, Garcia J, Klimstra DS. Pancreatic acinar cell carcinomas with prominent ductal differentiation: mixed acinar ductal carcinoma and mixed acinar endocrine ductal carcinoma. Am J Surg Pathol. 2010;34(4):510-518.

2. Kyriazi MA, Arkadopoulos N, Stafyla VK, et al. Mixed acinarendocrine carcinoma of the pancreas: a case report and review of the literature. Cases J. 2009;2:6481.

3. Farrell JM, Pang JC, Kim GE, Tabatabai ZL. Pancreatic neuroendocrine tumors: accurate grading with Ki-67 index on fine-needle aspiration specimens using the WHO 2010/ENETS criteria. Cancer Cytopathol. 2014;122(10):770-778.

4. Ohike N, Kosmahl M, Klöppel G. Mixed acinar-endocrine carcinoma of the pancreas. A clinicopathological study and comparison with acinar-cell carcinoma. Virchows Arch. 2004;445(3):231-235.

5. Yu R, Jih L, Zhai J, et al. Mixed acinar-endocrine carcinoma of the pancreas: new clinical and pathological features in a contemporary series. Pancreas. 2013;42(3):429-435.

6. Lee L, Bajor-Dattilo EB, Das K. Metastatic mixed acinar-neuroendocrine carcinoma of the pancreas to the liver: a cytopathology case report with review of the literature. Diagn Cytopathol. 2013;41(2):164-170.

7. Ogbonna OH, Garcon MC, Syrigos KN, Saif MW. Mixed acinarneuroendocrine carcinoma of the pancreas with neuroendocrine predominance. Case Rep Med. 2013;2013:705092.

8. Yantiss RK, Chang HK, Farraye FA, Compton CC, Odze RD. Prevalence and prognostic significance of acinar cell differentiation in pancreatic endocrine tumors. Am J Surg Pathol. 2002;26(7):893-901.

9. Hruban RH, Pitman MB, Klimstra DS. Tumors of the Pancreas. Vol 6. Washington, DC: Armed Forces Institute of Pathology: American Registry of Pathology; 2007.

10. Imaoka H, Amano Y, Moriyama I, Itoh S, Yanagisawa A, Kinoshita Y. Endoscopic ultrasound-guided fine-needle aspiration of a mixed acinarendocrine carcinoma: a case report. Am J Gastroenterol. 2008;103(10): 2659-2660.

11. Klimstra DS, Rosai J, Heffess CS. Mixed acinar-endocrine carcinomas of the pancreas. Am J Surg Pathol. 1994;18(8):765-778.

12. Mizuno N, Naruse S, Kitagawa M, et al. Insulinoma with subsequent association of Zollinger-Ellison syndrome. Intern Med. 2001;40(5): 386-390.

13. Kobayashi S, Asakura T, Ohike N, et al. Mixed acinar-endocrine carcinoma of the pancreas with intraductal growth into the main pancreatic duct: report of a case. Surg Today. 2010;40(4):380-384.

14. Capelli P, Martignoni G, Pedica F, et al. Endocrine neoplasms of the pancreas: pathologic and genetic features. Arch Pathol Lab Med. 2009; 133(3):350-364.
15. Klimstra DS. Nonductal neoplasms of the pancreas. Mod Pathol. 2007; 20 Suppl 1:S94-S112.

16. Kim MJ, Jang SJ, Yu E. Loss of E-cadherin and cytoplasmic-nuclear expression of beta-catenin are the most useful immunoprofiles in the diagnosis of solid-pseudopapillary neoplasm of the pancreas. Hum Pathol. 2008;39(2):251-258.

17. Guo Y, Yuan F, Deng H, Wang HF, Jin XL, Xiao JC. Paranuclear dotlike immunostaining for CD99: a unique staining pattern for diagnosing solid-pseudopapillary neoplasm of the pancreas. Am J Surg Pathol. 2011; 35(6):799-806.

18. Sullivan PS, Clebanoff JL, Hirschowitz SL. Hints to the diagnosis of mixed acinar-endocrine carcinoma on pancreatic fine-needle aspiration: avoiding a potential diagnostic pitfall. Acta Cytol. 2013;57(3): 296-302.

19. Wisnoski NC, Townsend CM Jr, Nealon WH, Freeman JL, Riall TS. 672 patients with acinar cell carcinoma of the pancreas: a populationbased comparison to pancreatic adenocarcinoma. Surgery. 2008;144(2): 141-148.

20. Rishi A, Goggins M, Wood LD, Hruban RH. Pathological and molecular evaluation of pancreatic neoplasms. Semin Oncol. 2015;42(1):28-39.

21. Soubra A, Faraj W, Saab J, Shamseddine A. Peri-ampullary mixed acinar-endocrine carcinoma. Rare Tumors. 2011;3(2):e15.

22. Chung WJ, Byun JH, Lee SS, Lee MG. Imaging findings in a case of mixed acinar-endocrine carcinoma of the pancreas. Korean J Radiol. 2010; 11(3):378-381.

23. Skacel M, Ormsby AH, Petras RE, McMahon JT, Henricks WH. Immunohistochemistry in the differential diagnosis of acinar and endocrine pancreatic neoplasms. Appl Immunohistochem Mol Morphol. 2000;8(3): 203-209.

24. Ogawa T, Isaji S, Yabana T. A case of mixed acinar-endocrine carcinoma of the pancreas discovered in an asymptomatic subject. In J Pancreatol. 2000;27(3):249-257.

25. Frank M, Bittinger A, Rothmund M, Arnold R. Immunohistochemical analysis and clinical course of high-malignant composite endocrineacinar cell carcinoma: a case report. Pancreas. 1998;17(2):210-212.

26. Shimoike T, Goto M, Nakano I, et al. Acinar-islet cell carcinoma presenting as insulinoma. $J$ Gastroenterol. 1997;32(6):830-835.

27. Cho KJ, Kim JY, Lee SS, Khang SK, Kim CW. Mixed acinar-endocrine carcinoma of the pancreas - a case report. J Korean Med Sci. 1996;11(2): 188-192.

28. Ichijima K, Akaishi K, Toyoda N, et al. Carcinoma of the pancreas with endocrine component in childhood. A case report. Am JClin Pathol. 1985; 83(1):95-100.

29. Ulich T, Cheng L, Lewin KJ. Acinar-endocrine cell tumor of the pancreas. Report of a pancreatic tumor containing both zymogen and neuroendocrine granules. Cancer. 1982;50(10):2099-2105. 


\section{Supplementary materials}

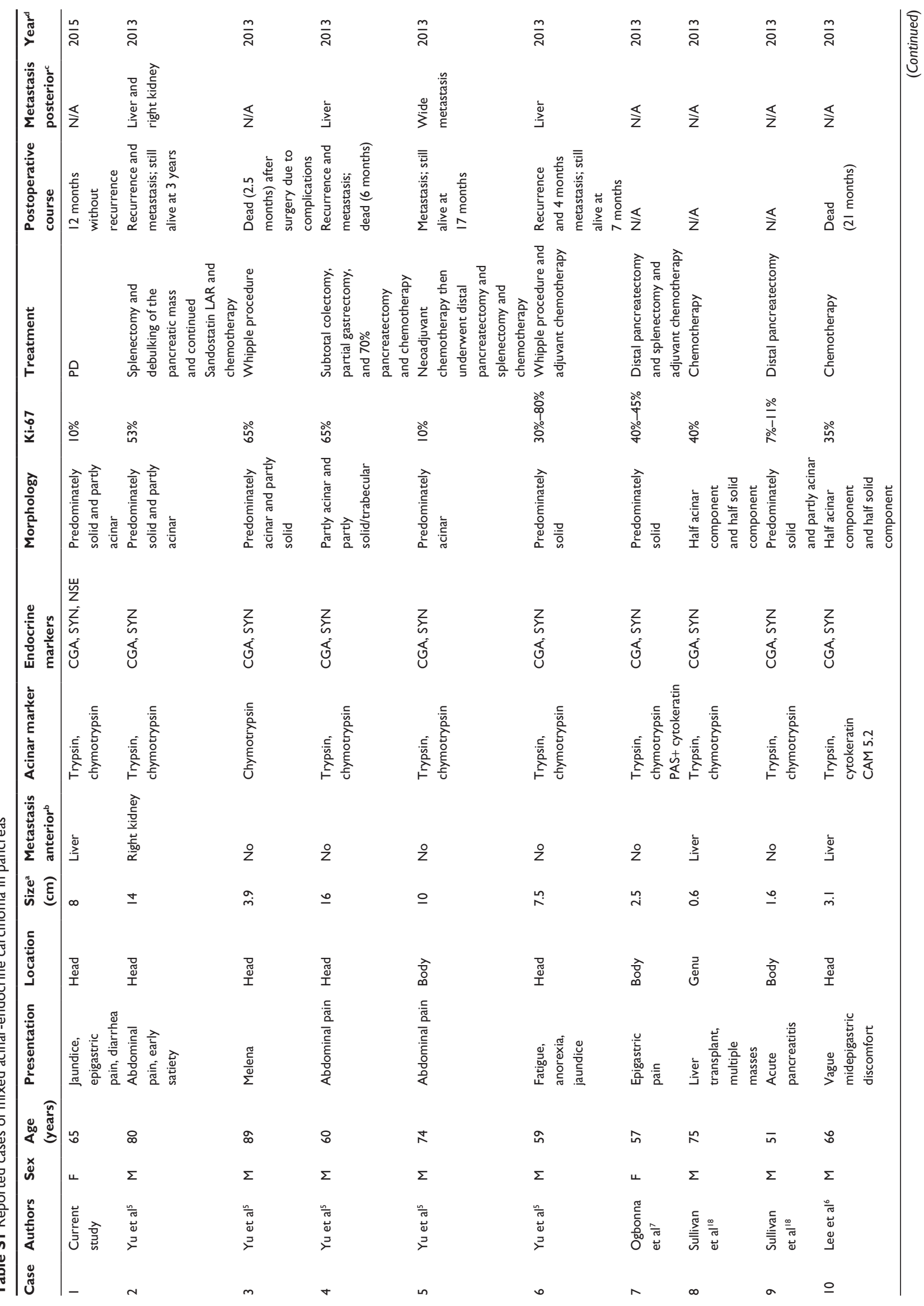




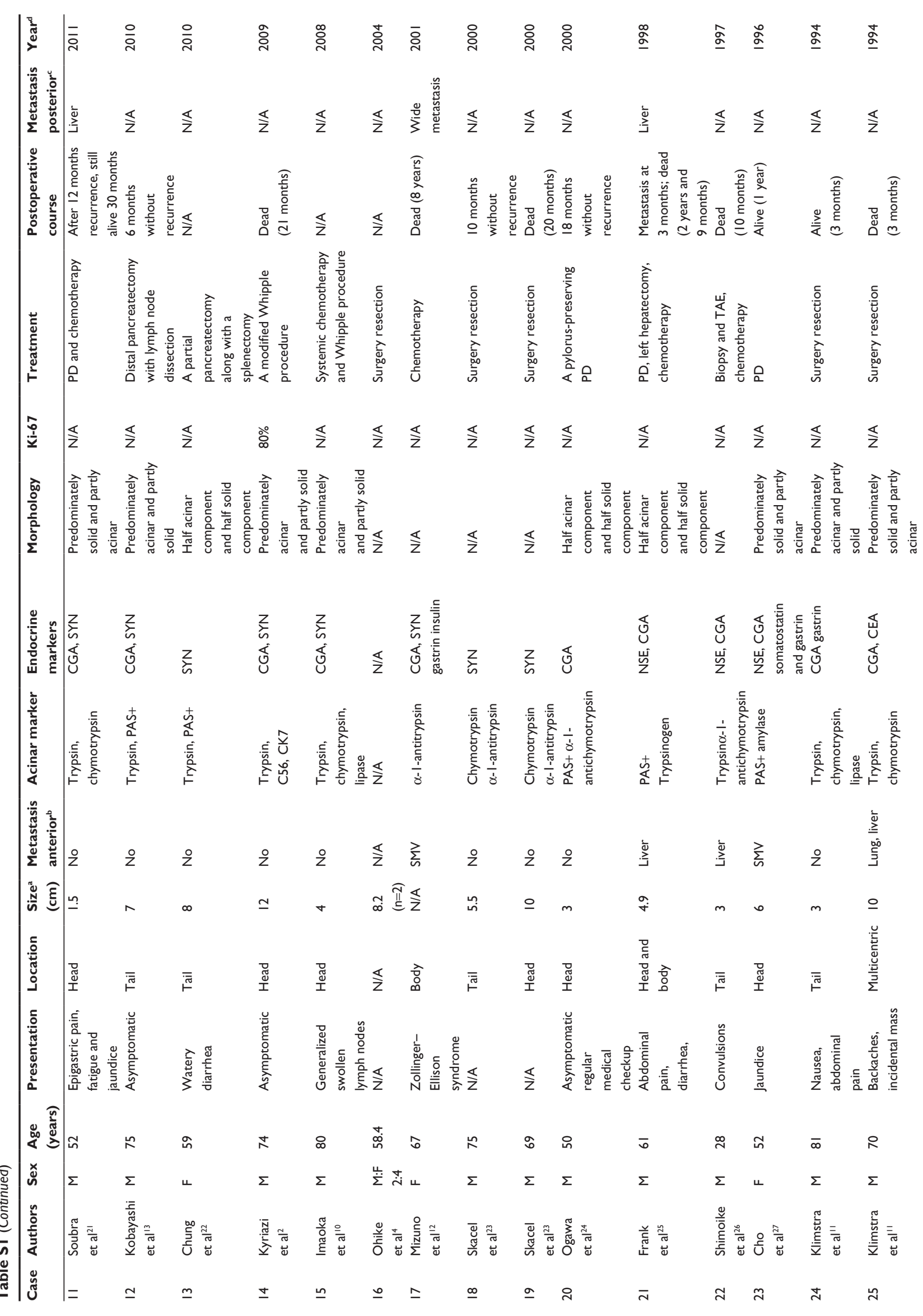




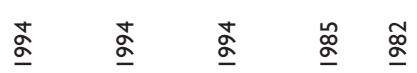

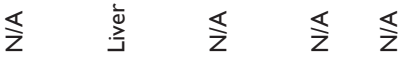

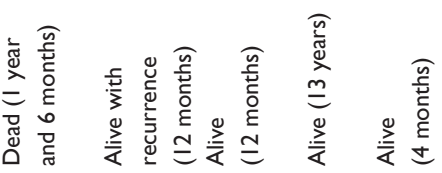

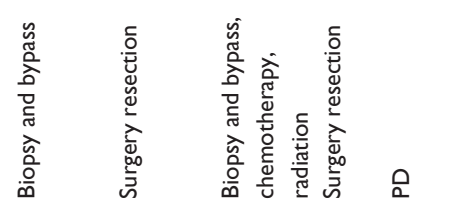

$\begin{array}{lllll}\frac{\pi}{z} & \frac{\pi}{z} & \frac{\pi}{z} & \frac{\pi}{z} & \frac{\pi}{z}\end{array}$

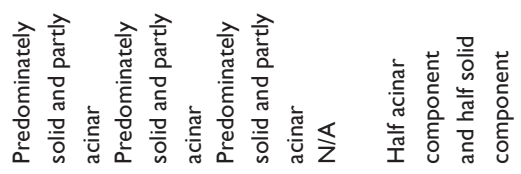

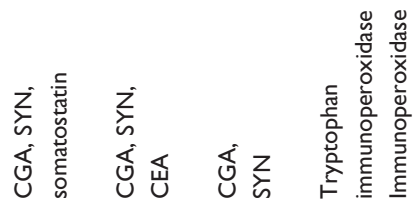

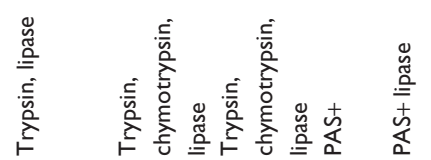

$\stackrel{\circ}{z} \quad \stackrel{0}{z} \quad \stackrel{0}{z} \quad \stackrel{0}{z} \quad \stackrel{2}{z}$

$\stackrel{\circ}{\circ} \infty \infty^{m}$

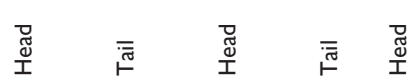

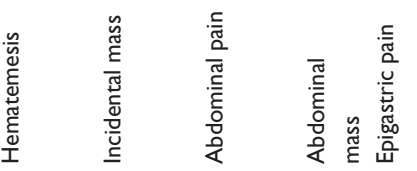

\&

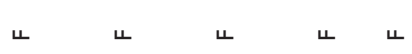

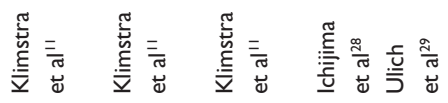

กิ

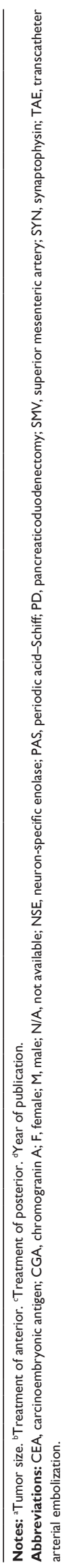

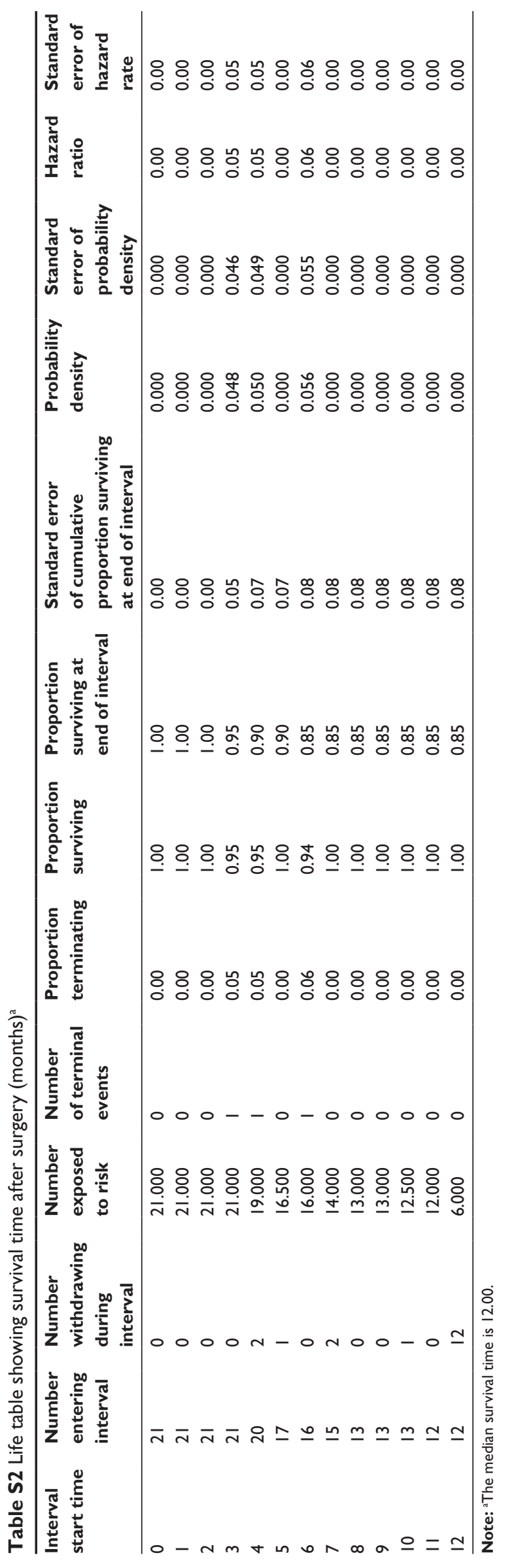




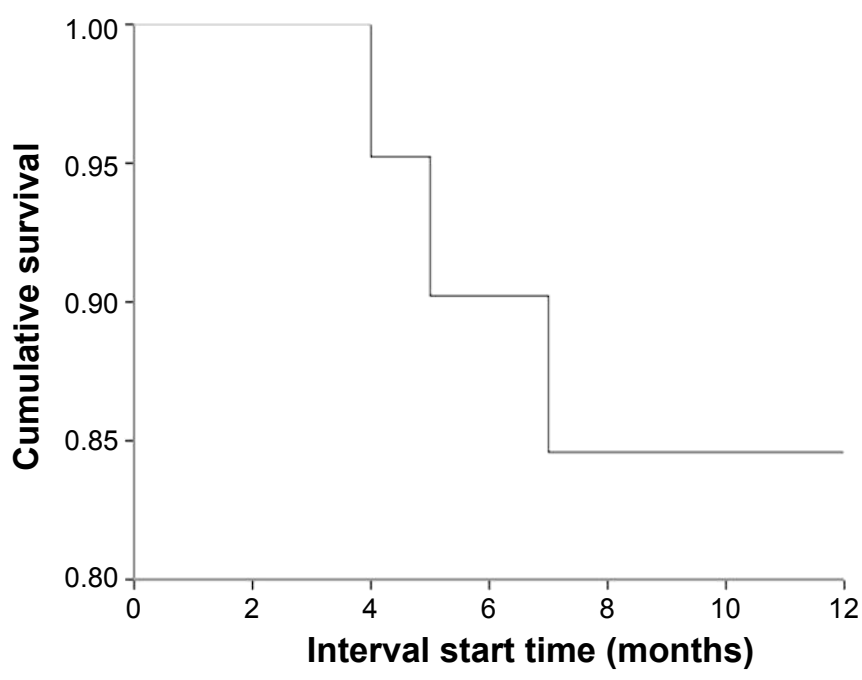

Figure SI Survival curve for the patients after surgery.

\section{Publish your work in this journal}

OncoTargets and Therapy is an international, peer-reviewed, open access journal focusing on the pathological basis of all cancers, potential targets for therapy and treatment protocols employed to improve the management of cancer patients. The journal also focuses on the impact of management programs and new therapeutic agents and protocols on patient perspectives such as quality of life, adherence and satisfaction. The manuscript management system is completely online and includes a very quick and fair peer-review system, which is all easy to use. Visit http://www.dovepress.com/testimonials.php to read real quotes from published authors. 